\title{
Contrasting Emergence: in Systems of Systems and in Social Networks
}

\section{Bernard P. Zeigler}

In the last note [1], I reviewed the notions of positive and negative emergence in the context of systems of systems, offering a tri-layered architecture to suggest how positive emergence might be promoted. The healthcare arena was offered an example where a better system of care delivery might emerge by coordinating the various systems and services currently providing care. This note will follow-up by examining a social media phenomenon -- the emergence of topics in the Twitter user community ${ }^{1}$. This has been proffered in a recent article [3] as an instance of emergence in the context of complex systems. Besides relating to contemporary social media, the article is thought-provoking as one of the first to take a modeling and simulation approach to emergence in social media. In particular, the tri-layered architecture and the associated conditions provide a definitive standard to assess such a putative instance of emergence. Moreover, such an examination might test the proposed abstraction itself, clarifying it, and suggesting improvements in it.

First, let's review the main contribution of the article [3], a study of how topics such as climate change grow in popularity among Twitter users (technically, micro-bloggers). The study uses Big Data techniques to cull nearly a million tweets on four popular topics from the Twitter Streaming API over a period of time and seeks to quantify the dynamics of topic popularity over this period. Advocates of these topics (frequent tweeters, both pro and con) and followers were identified and their tweeting behavior modeled, focusing especially on the propensity to retweet and spread awareness to new users. Using the data, parameter values were identified that enabled simulations in which the model is employed in Monte Carlo manner to generate the aggregate interest in a topic over time. The intensity of interest in a topic is defined as a metric involving the proportional number of tweets devoted to the topic and the proportional number of blogger participants devoted to it relative to all users. Perhaps, not surprisingly, topic popularity behaviors are found to follow trajectories over time characterized by the acronym: RPBS Rising, Plateau, Burst, and Stabilization, where a Markov model treating these as states provides probabilities of transitions mostly reflecting increasing intensity but with possible reversals.

Review of Before proceeding, let's review the tri-layered architectural framework from [1] (repeated here, for convenience, Figure 1).

\begin{tabular}{|c|}
\hline Coordination Economics \\
\hline Pragmatic Level Communication Network \\
\hline System of Systems Ecology \\
\hline
\end{tabular}

Figure 1. Tri-layered Architecture for Positive Emergence

\footnotetext{
${ }^{1}$ According to Wikipedia[2], "Twitter is an online social networking service that enables users to send and read short 140-character messages called "tweets". A social networking service (also social networking site or SNS) is a platform to build social networks or social relations among people who share similar interests, activities, backgrounds or real-life connections. Users access Twitter through the website interface, SMS or mobile device application."
} 
The layers were roughly characterized as follows:

- System of Systems Ecology (Primeval SoS): the systems that will become component systems of the emergent SoS already exist as viable autonomous entities in an ecology; however left unperturbed they would not emerge into the SoS under consideration.

- Network supporting Pragmatic level of communication: the ability to communicate among putative component systems of the SoS, not only at technical level, but a level that supports the coordination needed at the next level (this is the "Pragmatic" level vs the underlying syntactic and semantic layers $[4,5])$.

- Coordination Economics: a compound term we introduce to refer to 1) the coordination required to enable the components to interact in a manner that allows emergence of the SoS with its own purposes, and 2) the economic conditions that enable emergence - the collective benefit that the SoS affords versus the cost to individuals in their own sustainability to contribute to the SoS objectives.

I will place the Twitter emergence case in the same framework as the example of emergence in National Healthcare Systems [1] in the form of Table 1.

\begin{tabular}{|l|l|l|}
\hline $\begin{array}{l}\text { Component } \\
\text { Autonomous } \\
\text { Systems }\end{array}$ & Healthcare in US & Twitter Topic Dynamics \\
\hline $\begin{array}{l}\text { Component } \\
\text { Interactions }\end{array}$ & $\begin{array}{l}\text { Payers pay for services } \\
\text { provided to patients }\end{array}$ & $\begin{array}{l}\text { Tweet messages exchanged among } \\
\text { participants }\end{array}$ \\
\hline $\begin{array}{l}\text { Basis for } \\
\text { economic } \\
\text { survival }\end{array}$ & Mainly fee-for-service & Independent of posts \\
\hline $\begin{array}{l}\text { Communication } \\
\text { platform at } \\
\text { technical levels }\end{array}$ & $\begin{array}{l}\text { Snail mail, phone service, } \\
\text { Internet, electronic records }\end{array}$ & $\begin{array}{l}\text { Users access Twitter through the } \\
\text { website interface, SMS or mobile } \\
\text { device application. }\end{array}$ \\
\hline $\begin{array}{l}\text { Communication } \\
\text { platform at } \\
\text { pragmatic level }\end{array}$ & $\begin{array}{l}\text { Health information } \\
\text { exchange (Not yet } \\
\text { established nation-wide.) }\end{array}$ & $\begin{array}{l}\text { Online social networking service is a } \\
\text { platform to build social relations } \\
\text { among users who share similar } \\
\text { interests, activities, backgrounds or } \\
\text { real-life connections, enables users } \\
\text { to exchange 140-character "tweets". }\end{array}$ \\
\hline
\end{tabular}




\begin{tabular}{|l|l|l|}
\hline $\begin{array}{l}\text { Coordination } \\
\text { required }\end{array}$ & $\begin{array}{l}\text { Care Delivery Chains, } \\
\text { Pathway-based coordination }\end{array}$ & $\begin{array}{l}\text { Platform provides topic naming (via } \\
\text { hashtag), following and retweeting } \\
\text { mechanisms }\end{array}$ \\
\hline $\begin{array}{l}\text { Favorable } \\
\text { Economic } \\
\text { conditions }\end{array}$ & $\begin{array}{l}\text { Value-based purchasing, } \\
\text { fee-for-performance }\end{array}$ & N/A (see text) \\
\hline
\end{tabular}

Table 1. Comparison of Conditions for Emergence in Twitter and Healthcare

At the lowest layer, the existence of autonomous component systems, both Healthcare and Twitter are comparable, in that both are constituted by such entities. However, their relationship to the economic and coordination conditions of the highest layer diverge radically. It is true that Twitter supports the conditions necessary for users to coordinate on topic creation and intensity. However, the users are not economically dependent on the emergence of a topic in the way that healthcare providers, consumers, and payers are critically linked for their economic survival to the successful transition from uncoordinated to coordinated system. This is a sign that the positive emergence characterized by the tri-layered architecture is not descriptive of the type of emergence exemplified by that of Twitter topics. To proceed we will argue that Twitter emergence is a weak form of emergence and the stark contrast with the healthcare case illuminates the tri-layer conditions.

\section{Emergence of Topics in Twitter}

Reference [3] distinguish two kinds of emergence, one related to properties and the other to networks. They refer to emergent properties as properties of systems that that are irreducible to the behavior of their components. A necessary condition seems to be that such emergence can only be manifest in systems with a large number of components and complex and dynamic interconnections. Recall that emergent properties that have undesired and unpredictable effects and consequences were referred to as negative emergence in the last note [1]. Emergence in social networks has been defined as the widespread adoption of a norm or convention such as use of a particular taxonomy to categorize topics by using hashtags or certain keywords. Taxonomies created by users are emergent processes whereby users collaborate to categorize topics or things using freely chosen keywords [3]. More relevant is the definition concerning the appearance and popularity of topics when a community adopts a particular means of expression by adopting a hashtag or keyword [7].

Rather than engage in a lengthy unpacking of such mystery-laden concepts as "irreducible" and "complex" we note that in our architecture, there is no mention of reducibility since we are not concerned with the emergence of properties but rather of a new system from existing system components. Further there is no mention of the number and complexity of component systems in the primeval ecology from which the SoS emerges. Thus there is no necessary reliance on large numbers of people, such as in social networks, to collaborate for a topic to emerge. In contrast with large social networks, we consider that a small collection of physicians could form an Integrated Practice Unit (IPU) as an instance of a positive emergence [7]. This brings out the conditions required by the architecture that the components are initially not bonded to the end state SoS and that they join together to form this system by relinquishing an existing mode of economic survival in favor of the new regime the emergent system fosters. In the case of the IPU, physicians initially owning their own practice band together to form the IPU and give up their own self-directed treatment of 
patients to conform to the needs of the larger collective with the benefit of increased cost effectiveness and financial viability. None of this is intended to exclude the case of large numbers of component systems or cardinalities of their states as typically being characteristic of the primordial SoS. Indeed, this is typically the case, and drives us toward simulation rather than analysis to understand how the conditions of the architecture can be realized.

\section{Emergence Monitoring and Detection}

Although emergence of topics in Twitter is a different kind of emergence, the study of intensity dynamics suggests a parallel to the need for Emergence Behavior Observers (EBO) to detect the emergence in the SoS context. As described by Mittal and Rainey [8], these offer services for querying component-states and interactions in real time from executing simulations that give heads up on impending emergence. Figure 2a) summarizes the methodology employed by Birdsey et al [3] to develop a user behavior model and employ it to do "what if" simulations of the user community in emergence of topics. Figure $2 b$ ) casts this methodology into a design approach for EBOs. Here we construct a dynamic model of the primordial SoS with the intent to employ it to predict where critical conditions might be developing to implicate emergence of a new SoS. We assume that the primordial SoS has not been well modeled or that such models exist but are too resource-demanding to run in real time. In either case, we employ (big) datadriven techniques to generate dynamic partial models targeted to aspects of emergence of interest and which run fast enough to extrapolate ahead from observed trajectories.

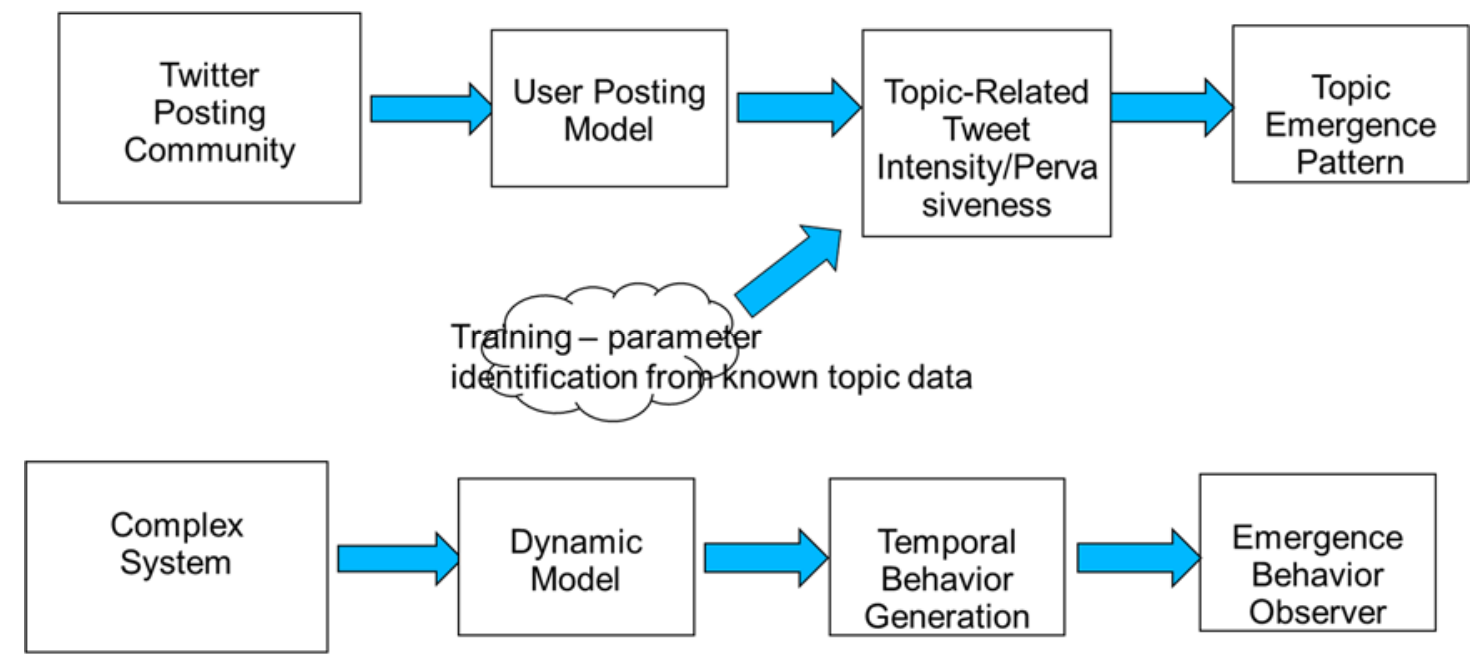

Figure 2. Detecting topic emergence as a guide to EBO design

In their ground-breaking paper, Mittal and Rainey [8] integrate the EBO concept into support for sustaining an emergent behavior through control policies. They offer six cybernetic principles to transform EBO outputs into a model that can be understood and scrutinized by humans. These principles are directly applicable to Figure $2 b$ ) suggesting how its Twitter-inspired methodology can be fleshed out and included within a full-capability cloud-based simulation environment. I hope to take this up in more detail in future notes.

\section{Conclusion}


Mittal [9] indicated two types of SoS: one that emerges from the bottom-up and the other that is desired from a top-down systems engineering approach. Emergence of topics in Twitter provides a clear example of bottom up emergence and (to the extent that the tri-layer architectural conditions are satisfied) is suggestive of how a SoS can come into existence. The architecture for promoting positive emergence from a top-down perspective is intended to be applicable to socio-technical systems of systems generally. Although perhaps deceptively simple, it is intended to provide the right core abstraction that can be refined and extended as needed in the intended domain application. It can be combined with Emergence Behavior Observers that can identify emergence of structures in advance, reducing their potential to surprise. Here we extended the breath of application of EBOs to include detecting the inception and temporal evolution of emergent SoSs. Although this methodological conception was inspired from emergence of topics in Twitter, we demonstrated that critical conditions proposed in the tri-layered architecture are not satisfied in the latter case. Specifically, the existing system components have survival at stake in emerging from the primordial socio-technological SoS but not in topic emergence. Still, there are useful analogies to be drawn from the former phenomenon. New topics can appear at any time and detecting when and how they arise to popular attention and stabilize offers potential insight into emergence of a new SoS. Modeling Emergence Behavior Observers after the topic emergence models suggests a potential approach to their design and implementation. The inclusion of both trending and waning in the RPBS model allows distinguishing major fluctuations in topic pervasiveness from statistical fluctuations better characterized as noise. The inclusion of stabilization also might be instructive with the caveat that collaborators have much less to lose in one case than the other in breaking up. Finally, the desire of the Twitter authors [3] to deploy the RPBS model into a live Twitter stream parallels the real-time deployment in cloud environments that is envisioned for the EBO concept [8]. 
1. Bernard P. Zeigler," A note on promoting positive emergence and managing negative emergence in systems of systems," The Journal of Defense Modeling and Simulation: Applications, Methodology, Technology, P 133-136, doi 10.1177/1548512915620580,13,1, http://dms.sagepub.com/content/13/1/133.abstract

2. Wikipedia, Twitter, https://en.wikipedia.org/wiki/Twitter

3. Birdsey, L., C. Szabo, and Yong Meng Teo, Twitter Knows: Understanding The Emergence Of Topics In Social Networks, Proceedings of the 2015 Winter Simulation Conference, L. Yilmaz, W. K. V. Chan, I. Moon, T. M. K. Roeder, C. Macal, and M. D. Rossetti, eds

4. Zeigler, B. P. and P. Hammonds Modeling \& Simulation-Based Data Engineering: Introducing Pragmatics into Ontologies for Net-Centric Information Exchange, Academic Press, Boston, 2007.

5. Nutaro J. and P. Hammonds. Combining the Model/View/Control Design Pattern with the DEVS Formalism to Achieve Rigor and Reusability in Distributed Simulation. The Journal of Defense Modeling and Simulation, 1(1):19-28, April 2004.

6. Yang, J. and J. Lescovec, Modeling Information Diffusion in Implicit Networks, IEEE International Conference On Data Mining (ICDM), 2010, 599-608

7. Zeigler, B. P., "Discrete Event System Specification Framework for Self-Improving Healthcare Service Systems," to appear in IEEE Systems Jnl

8. Mittal, S. and L. Rainey, Harnessing Emergence: The Control and Design and Emergent Behavior in System of Systems Engineering SummerSim, 2015

9. Mittal, S. Emergence in stigmergic and complex adaptive systems: A formal discrete event systems perspective, Cognitive Systems Research archive, Volume 21, March, 2013, Pages 22-39 doi $>10.1016 /$ j.cogsys.2012.06.003 
